\title{
Why are there eccentricity effects in visual search? Visual and attentional hypotheses
}

\author{
JEREMY M. WOLFE and PATRICIA O'NEILL \\ Harvard Medical School, Boston, Massachusetts \\ and Brigham and Women's Hospital, Boston, Massachusetts \\ and \\ SARA C. BENNETT \\ Brigham and Women's Hospital, Boston, Massachusetts
}

\begin{abstract}
In standard visual search experiments, observers search for a target item among distracting items. The locations of target items are generally random within the display and ignored as a factor in data analysis. Previous work has shown that targets presented near fixation are, in fact, found more efficiently than are targets presented at more peripheral locations. This paper proposes that the primary cause of this "eccentricity effect" (Carrasco, Evert, Chang, \& Katz, 1995) is an attentional bias that allocates attention preferentially to central items. The first four experiments dealt with the possibility that visual, and not attentional, factors underlie the eccentricity effect. They showed that the eccentricity effect cannot be accounted for by the peripheral reduction in visual sensitivity, peripheral crowding, or cortical magnification. Experiment 5 tested the attention allocation model and also showed that RT $\times$ set size effects can be independent of eccentricity effects. Experiment 6 showed that the effective set size in a search task depends, in part, on the eccentricity of the target because observers search from fixation outward.
\end{abstract}

In laboratory visual search tasks, subjects look for a target item among some number of distracting items. In most cases, the positions of targets and distractors are random across trials. This makes the implicit assumption that, within reason, it does not matter where the targets and distractors fall in the visual field. No doubt, any researcher in the field, if queried, would agree that this cannot be strictly true. It seems intuitively clear-and it has been experimentally demonstrated-that targets presented at the point of fixation are likely to be detected and localized more quickly and accurately than are targets presented even in the near periphery (Carrasco \& Chang, 1995; Carrasco, Evert, Chang, \& Katz, 1995). In this paper, we argue for a simple, attentional account for eccentricity effects in visual search. This account is an elaboration of the Guided Search model of visual search (Wolfe, 1994; Wolfe, Cave, \& Franzel, 1989; Wolfe \& Gancarz, 1996). Guided Search holds that attention in visual search tasks is deployed to one item at a time, and it can be redeployed at a rate of one item approximately every 50 msec (Bennett \& Wolfe, 1996; Wolfe \& Gancarz,

This research was supported by NIH-NEI EYO5087 and AFOSR 49620-93-1-0407 to J.M.W. and by a NIH-NRSA Postdoctoral Fellowship to P.O. The authors thank Greg Gancarz, Todd Horowitz, Kari Dahlen, Nikki Klempen, Jan Theeuwes, Marisa Carrasco, and Asher Cohen for comments on drafts of this paper. P. O'Neill is now at the University of Mississippi. Correspondence should be addressed to J. M. Wolfe, Harvard Medical School, Brigham \& Women's Hospital, Department of Ophthalmology, 221 Longwood Ave., Boston, MA 02115 (e-mail: wolfe@search.bwh.harvard.edu).
1996). The order in which items are attended is determined by their activation. Items receive more activation if they share features with the designated target. Thus, if one is searching for the red vertical line, red items gain activation and vertical items gain activation. These items will be attended to before items that are neither red nor vertical. Here, we add one assumption to that scheme: Activation is modulated by eccentricity. Given two identical items at different distances from fixation, the item closer to fixation will receive more activation and will be attended before the more eccentric item. Since central locations have a greater representation in the visual system than have peripheral locations, it is reasonable to imagine that central items produce a bigger attentionguiding signal than do peripheral items.

There is an alternative explanation of eccentricity effects in search: They could be due to direct effects of eccentricity on visual performance (e.g., Carrasco \& Chang, 1995; Carrasco et al., 1995; Geisler \& Chou, 1995). It could take longer to find a target in the periphery because items are harder to see in the periphery. In this paper, we will present data that argue for an attentional component to eccentricity effects separate from any purely visual effect.

A variety of studies have been done on the effects of target position in visual search or search-like tasks. Numerous investigators have noted that search is harder for targets in the periphery of the visual field (e.g., Bursill, 1958; Cole \& Hughes, 1984; Efron, Yund, \& Nichols, 1990; Lee, Jung, \& Chung, 1992; Previc \& Blume, 1993; Remington \& Williams, 1986; Saarinen, 1993; Sanders, 1970; Sanders \& Brück, 1991). The most systematic work 
on the effects of eccentricity has been done by Carrasco and her colleagues (Carrasco \& Chang, 1995; Carrasco \& Evert, 1991; Carrasco et al., 1995; Carrasco \& Katz, 1992). They have demonstrated the impact of visual factors, such as cortical magnification (Carrasco \& Frieder, 1997), spatial resolution (Carrasco, McLean, \& Frieder, in press) and lateral interactions (Carrasco \& Chang, 1995), and have argued for a visual account of eccentricity effects. In addition, they argue that eccentricity effects are the underlying cause of some reaction time (RT) $\times$ set size effects (see also Geisler \& Chou, 1995). This is an important claim because RT $\times$ set size functions are central to most theoretical accounts of visual search (Duncan \& Humphreys, 1989; Treisman, 1993; Treisman \& Gelade, 1980; Wolfe, 1994; Wolfe et al., 1989). Furthermore, much of the analysis of visual search is based on the assumption that eccentricity effects can be ignored in the analysis of those set size effects.

Other studies have argued against a close link between low-level visual resolution and the eccentricity effect in visual search. Ball, Owsley, and Beard (1990) found that their measure of the "useful field of view" (UFOV) was dissociable from standard measures of visual fields (Ball, Owsley, \& Beard, 1990). The UFOV is a visual field isopter derived from accuracy measures on a set of tasks that include a visual search component. To a first approximation, the UFOV defines a visual field inside of which subjects can do a task and outside of which they cannot. The idea has its roots in, for example, Sanders's (1970) work on the functional field of view, the part of the visual field that can be used during a single fixation. Ball and her colleagues report that the UFOV is smaller in the old than in the young (Ball, Beard, Roenker, Miller, \& Griggs, 1988), shrinks in the presence of cognitive load (Graves et al., 1993), and correlates with automobile accidents (Ball, Owsley, Sloane, Roenker, \& Bruni, 1993). While the experimental design is different from the usual visual search experiment, the UFOV work is interesting because it demonstrates correlations between eccentricity effects and real-world variables, such as the incidence of traffic accidents (Ball et al., 1993). An inability to attend to an object in the periphery can have consequences comparable to an inability to see an object in the periphery (see also Ikeda \& Takeuchi, 1975; Weltman \& Egstrom, 1966; Williams, 1982, 1985).

Other studies point to a distinction between eccentricity effects in attention and visual resolution. Cole and Hughes (1984) found that the eccentricity effect was not eliminated when the size of the peripheral targets was increased to compensate for the decline in visual acuity and sensitivity with eccentricity. Moreover, such variables as age (Ball, Roenker, \& Bruni, 1990; Madden, 1992; Scialfa, Kline, \& Lyman, 1987), mental load (Egeth, 1977; Logan, 1978), and stress (Bursill, 1958) all have an impact on search performance that seems to be largely independent of their effects on such measures as acuity. These studies, like the UFOV studies, use tasks that are quite different from the standard search tasks in which an observer searches for a target item among a variable number of distractor items.

Our experiments were intended to disentangle the visual and attentional accounts of eccentricity effects in visual search. In Experiment 1, we replicated the basic finding of Carrasco et al. (1995), showing that our particular methods produced a large and reliable eccentricity effect using standard visual search stimuli. The results of Experiment 2 argued that eccentricity effects in visual search are not a simple by-product of the decline in visual resolution and sensitivity with eccentricity. Experiment 3 manipulated the spacing of targets and distractors and showed that the eccentricity effects we observed were not dependent on increased lateral interactions or "crowding" in peripheral vision (e.g., Levi, Klein, \& Aitsebaomo, 1985). Experiment 4 showed that strong eccentricity effects can occur with stimuli that are scaled in size to compensate for cortical magnification of the central portions of the visual field (e.g., Wilson, Levi, Maffei, Rovamo, \& DeValois, 1990). (In this paper, after Experiment 4, the attentional account of eccentricity effects is described in more detail.) Experiment 5 argued that the theoretically important $\mathrm{RT} \times$ set size functions are not side effects of eccentricity effects. Eccentricity effects and set size effects can be discussed as theoretically distinct issues even though they can interact in the data. Finally, Experiments 5 and 6 tested predictions of the hypothesis that eccentricity effects represent a foveal bias in the allocation of attention.

\section{EXPERIMENT 1 The RT $\times$ Eccentricity Function}

Carrasco et al. (1995) have documented the existence of eccentricity effects in visual search. In Experiment 1, we replicated this basic finding in order to document the existence and size of the effect using our particular visual search stimuli. In this experiment, following Carrasco et al. (1995), we examined the effects of target position in a standard search for a conjunction of color $X$ orientation.

\section{Method}

There were two conditions in Experiment 1. In the one-target condition, the target was red $\left(11.2 \mathrm{~cd} / \mathrm{m}^{2}\right.$; CIE coordinates, $x=$ $0.618, y=0.353)$ and vertical $\left(1.3^{\circ} \times 0.3^{\circ}\right)$. In the two-target condition, the target could be either red vertical or green horizontal $\left(25.0 \mathrm{~cd} / \mathrm{m}^{2}\right.$; CIE coordinates, $\left.x=0.282, y=0.592\right)$. The background was gray $\left(5.0 \mathrm{~cd} / \mathrm{m}^{2}\right)$. In the two-target condition, target identity was random across trials. For $450 \mathrm{msec}$ at the start of each trial, either a red vertial line or a green horizontal line in a white box at the point of fixation specified the target for the trial. In both conditions, distractors were green vertical lines and red horizontal lines, so that the bulk of the display was the same in the two conditions. By running two conditions with essentially the same visual stimuli, we can begin to discriminate between the impact of visual factors and attentional/task demand factors on the eccentricity effect.

Stimuli were presented on a standard Macintosh computer monitor in a region that subtended $16^{\circ} \times 16^{\circ}$. Set sizes of 25,35 , and 45 items were used. We used relatively large set sizes in this experiment 
so that, even in a relatively efficient conjunction search task (Wolfe, 1992), attention would need to be deployed to several items in each search. Some caution is required in generalizing from one range of set sizes to another, since search behavior may change as a function of set size. For example, Carrasco et al. (1995) looked at a wide range of set sizes within a single experiment. They report some compressive nonlinearity in the $\mathrm{RT} \times$ set size functions, perhaps due to increased errors at the larger set sizes (see also Wolfe et al., 1989). In this paper, different set size ranges were used in different experiments, resulting in essentially the same pattern of eccentricity effects.

The $16^{\circ} \times 16^{\circ}$ screen was divided into a $7 \times 7$ array of cells. Distractors could appear in any cell except the cell at fixation. To reduce the numbers of trials per subject, targets were constrained to appear in the 24 locations forming eight arms radiating from fixation (see Figure 1).

Cutting the number of target locations in half saves hundreds of trials. Target locations were not shaded or otherwise indicated in the display seen by the subjects. The subjects did not know the distribution of target locations; even if they had known the distribution, there is no evidence that subjects can spread attention in the octopus shape required to exploit that information. (In other experiments, we have found that subjects are unable to bias attention to four locations defining a square even when they know the locations.)

Within the constraints just described, placement of targets and distractors was random from trial to trial. Targets were presented on $67 \%$ of the trials. (Blank trials are required to keep the subject "honest" but are relatively uninteresting in a study of target location.) Before each session, the task was described to the subject. The subjects were asked to respond as quickly as possible while keeping their error rate under $10 \%$. They were not informed about the ratio of target-present to target-absent trials. The subjects were tested for 30 practice trials followed by 300 experimental trials. Stimulus presentation and data collection were controlled by MacProbe software (Hunt, 1994).

Ten subjects between 18 and 46 years of age were tested. All had visual acuity of $20 / 25$ or better, and all could pass the Ishihara color

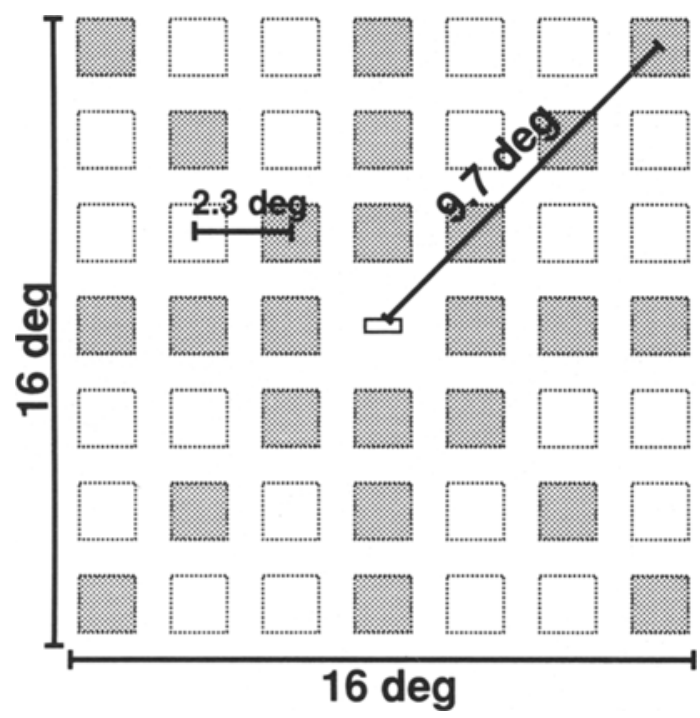

Figure 1. Stimulus configuration for Experiment 1. Distractors could appear in any of the 48 loci (excluding fixation). Targets were constrained to appear only in the shaded loci. Neither boxes nor shading was present in the actual stimulus display. test. The subjects gave informed consent, and they were paid for their time. Order of conditions was randomized across subjects.

\section{Results}

Mean RTs at each set size were used to generate the standard RT $\times$ set size functions. These are shown in Figure 2. The results for the one-target condition reveal the usual efficient search for color $X$ orientation conjunctions (Wolfe et al., 1989). Slopes of the RT $\times$ set size functions were $3.0 \mathrm{msec} /$ item for the target trial and $12.2 \mathrm{msec} / \mathrm{item}$ for the blank trials. The results of the two-target condition show that the slopes remained shallow ( 5.9 and $18.0 \mathrm{msec} /$ item, respectively), but that the mean RTs were significantly slower [paired $t(9)=10.5$, $p<.001$ ], replicating an earlier result from our lab (Wolfe, 1992). Error rates for the one-target condition were $2.2 \%$ for miss errors and $1.7 \%$ for false alarms (FAs). Error rates for the two-target condition were $5.2 \%$ for miss errors and $2.3 \%$ for FAs.

Figure 3 (upper panel) shows the average of the mean RTs for all subjects as a function of eccentricity. In practice, each point in the figure represents data averaged over four locations with the same eccentricity. Errors are shown in the lower panel of Figure 3.

Replicating the results of Carrasco et al. (1995), the results reveal a clear eccentricity effect. RTs increased as the target moved farther into the periphery. This is true for each condition in the experiment. The eccentricity effect was significantly greater for the two-target condition than for the one-target condition $[t(9)=5.17, p<$ $.001]$. Errors showed a moderate increase as the target moved to the periphery.

In the two-target condition, green-target trials took longer $(1,644 \mathrm{msec})$ than did red-target trials $(1,472 \mathrm{msec})$ $[F(1,9)=19.69, p<.002]$, and the subjects were more likely to miss the green target $(5.8 \%)$ than the red target $(1.9 \%)[F(1,9)=34.74, p<.001]$. The $\mathrm{RT} \times$ set size slopes were not significantly steeper for green targets $[t(9)=0.74, \mathrm{n} . \mathrm{s}$.$] , whereas the eccentricity functions$ were steeper $[t(9)=2.44, p<.019]$. Because of these differences between red and green targets, we thought that it would be instructive to compare the one-target condition with the red-target trials of the two-target condition. These one- and two-target trials were physically identical. The RT $\times$ set size slopes were still significantly steeper for the two-target condition $[t(9)=2.04, p<.036]$, and the eccentricity functions were still significantly steeper for the two-target condition $[t(9)=2.07, p<.031]$. Thus, the change in the task altered the set size and eccentricity slopes for stimuli that were otherwise identical in the one- and two-target conditions.

Visual field effects. The results as shown in Figure 3 are averaged across the upper and lower and the left and right portions of the visual field. To assess the visual field effects, R'Ts were pooled across subjects, and RTs from the upper field were compared with those from the lower field. Results for the one-target condition show that the RTs for the upper visual field were significantly shorter 
Target Trials

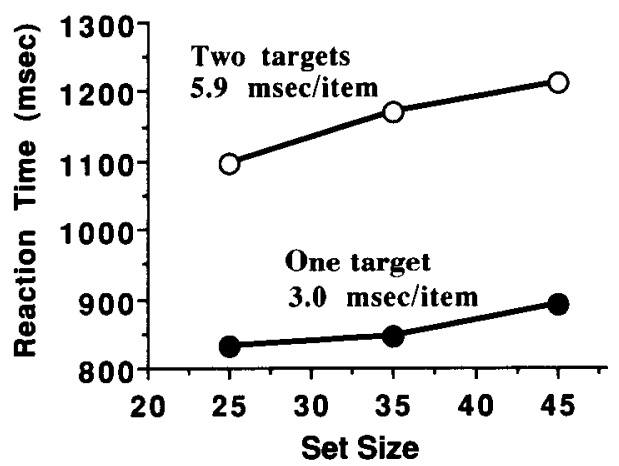

Blank Trials

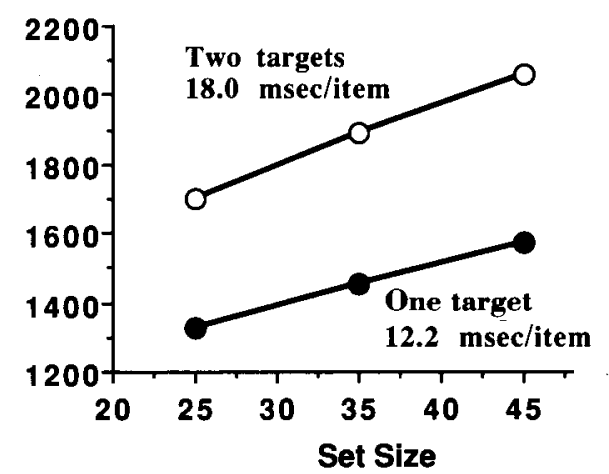

Figure 2. RT $\times$ set size functions for the two conditions of Experiment 1. Note that slopes were quite shallow for target-present trials in both conditions, although the RTs for the two-target condition were much slower than those for the one-target condition.

than the RTs from the lower visual field (mean RTs: upper field, $814 \mathrm{msec}$; lower field, $917 \mathrm{msec}$ ) $[F(1,9)=12.47$, $p<.01]$. Results for the two-target condition followed this pattern (mean RTs: upper field, $1,143 \mathrm{msec}$; lower field, $1,257 \mathrm{msec})[F(1,9)=8.70, p<.025]$. In the two-target condition, there were more errors in the lower field $(6.0 \%$ vs. $4.2 \%)$. As reported by others (Christman \& Naegele, 1995; Yund, Efron, \& Nichols, 1990), RTs from the right visual field were somewhat faster than those from the left, but these results did not quite rise to the .05 level of significance: one-target condition (right, $838 \mathrm{msec}$; left, $887 \mathrm{msec}$ ) $[F(1,9)=4.09, p<.1]$; twotarget condition (right, 1,169 msec; left, 1,219 msec) $[F(1,9)=3.08, p>.1]$.

Figure 4 shows median RTs and miss rates for each of the 24 target locations for the two-target condition of
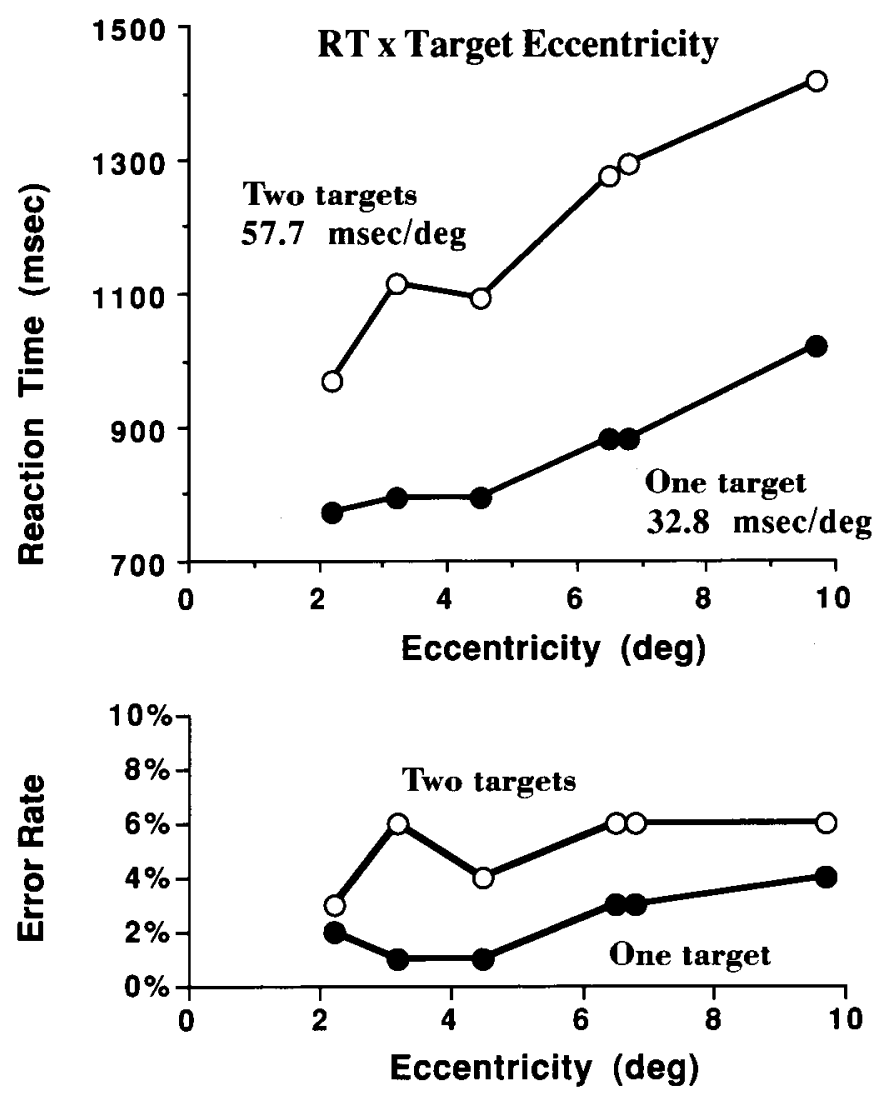

Figure 3. Eccentricity functions for Experiment 1. Upper panel: RT results. Lower panel: errors. 
Experiment 1 . This gives a more complete picture of the data, but it should be noted that individual cells contained relatively few trials. We did not examine the effects of eccentricity on RT $\times$ set size functions, because a very large number of trials is required. This was the subject of Experiment 6 (below).

\section{Discussion}

As should be clear from Figure 3, eccentricity effects are large, even in an efficient search such as the color $X$ orientation search used here. A mere $10^{\circ}$ from fixation, RTs are as much as several hundred milliseconds slower than they are at $2^{\circ}$ from fixation. If we assume that this roughly linear effect continues farther into the periphery, it is easy to imagine that eccentricity effects could have significant behavioral consequences for tasks that require speeded responses to stimuli at many locations in the visual field (e.g., driving). The apparent differences between the left and right and the upper and lower visual fields are interesting but are not central to the issues in this paper. Our emphasis will be on the effects of eccentricity. However, before abandoning the field differences, it is worth pointing out that the existence of significant differences between fields makes it hard to explain as a by-product of low-level constraints on visual resolution and/or sensitivity, since most of those constraints are roughly symmetrical with binocular viewing (Grigsby \& Tsou, 1994; Rovamo \& Virsu, 1979). An attentional account would have to propose a bias that favored the right field and the upper field. However, it would not be wise to theorize too heavily about these field differences. Yund et al. (1990) do not report upper versus lower field effects, and Carrasco et al. (1995, Table 5) report essentially no field effects. If the field effects are theoretically important, further data will be needed to better document them.

We can begin to disentangle the visual and attentional accounts of eccentricity effects by comparing the oneand two-target conditions of Experiment 1. The visual stimuli were virtually identical in the two conditions, but the eccentricity effects were quite different in magnitude. This was true even when the analysis of the twotarget data was restricted to the red targets that were identical to those used in the one-target condition. The visual stimuli were identical, the attentional demands differed, and the eccentricity effects differed. This would seem to argue for an attentional component to the eccentricity effect in this experiment. These results do not show that attention is the sole factor in producing eccentricity effects. We know that visual factors can produce eccentricity effects. For example, Geisler and Chou (1995) used small stimuli that would be hard to detect in the periphery, and

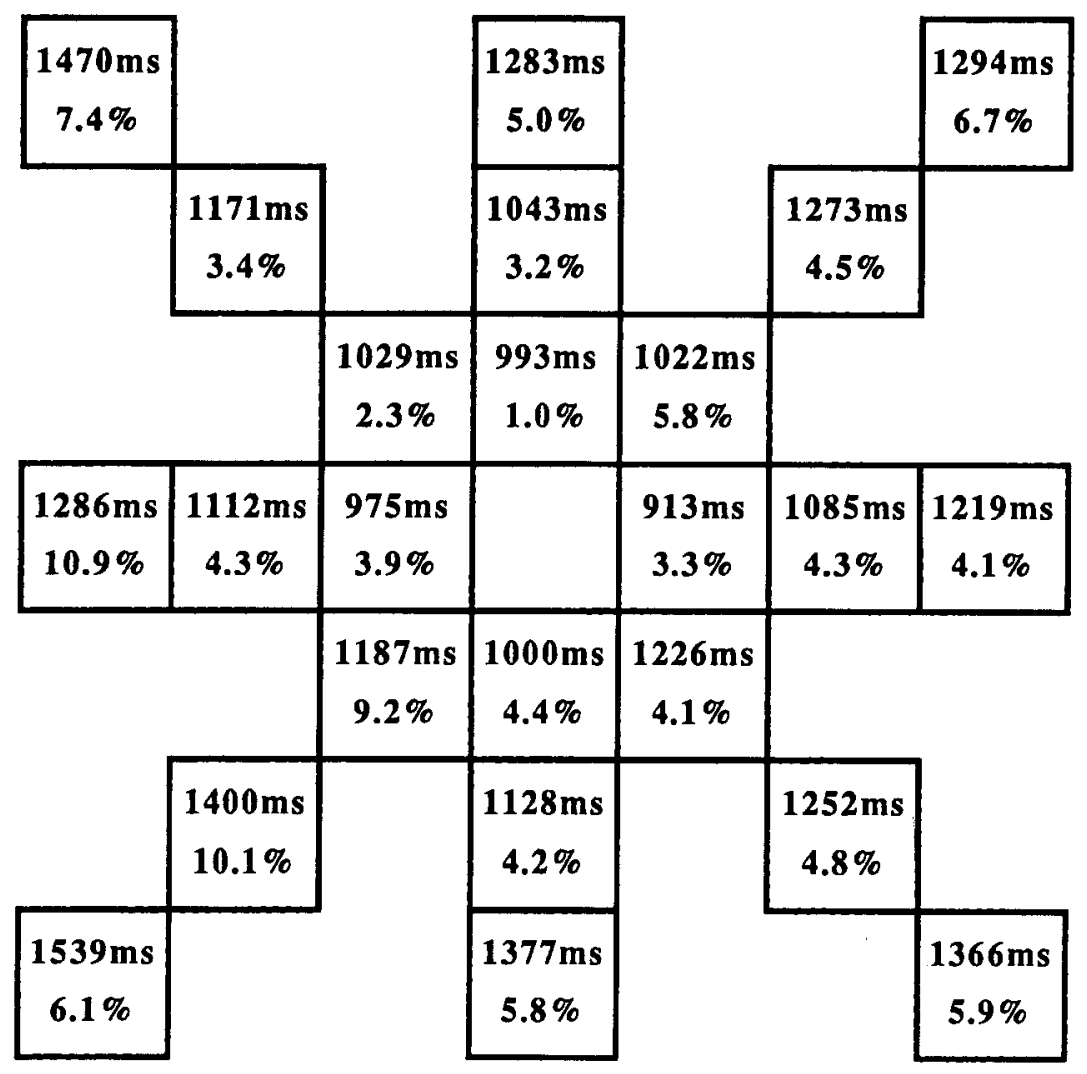

Figure 4. Median RTs (in milliseconds) and percent misses (\%) for each possible target location averaged over all subjects in the two-target condition of Experiment 1. 
they showed that "low-level" visual factors have an important impact on performance. The results of Experiment 1 suggest that, with our relatively large and saturated stimuli, these visual constraints are not the sole determinant of eccentricity effects. The results of Experiment 1 support the hypothesis that, like visual factors, allocation of attention varies with eccentricity. The next experiments further test this hypothesis.

\section{EXPERIMENT 2 Peripheral Resolution Effects}

Visual factors could produce slowed responses to peripheral visual search targets in a number of ways. For example, it might take several hundred milliseconds longer to decide on the identity of an item at $10^{\circ}$ than to decide on the identity of an item at $2^{\circ}$. If peripheral processing were simply slower than central processing, then it should be slower even if only a single item is presented. Accordingly, the two-target condition of Experiment 1 was rerun with only a single item on the screen. In effect, this was a speeded classification task with spatial uncertainty.

\begin{abstract}
Method
If the single item was red horizontal or green vertical, the subjects pressed one response key. If it was green horizontal or red vertical, they pressed the other. The two-target condition allowed us to check peripheral response to both the red vertical and the green horizontal targets. In addition, as a speeded classification task, the Set Size 1 two-target condition had the methodological advantage over the one-target condition of mapping two items to each response. All other properties of the stimulus and all other methods were identical to those described for the two-target condition of Experiment 1 . The same 10 subjects were tested, allowing direct comparison of search through multiple items and identification of a single item. The subjects were tested for 100 trials.
\end{abstract}

\section{Results and Discussion}

The results for Experiment 2 are plotted in Figure 5 together with the relevant comparison stimuli from Experiment 1 . They show that the eccentricity function for a set size of 1 was much shallower than the function for set sizes of 25, 35, and 45 items. Misses did not change significantly as a function of eccentricity $\left(2.3^{\circ}=3.5 \%\right.$ misses; $3.2^{\circ}=2.7 \%$ misses; $4.6^{\circ}=1.8 \%$ misses; $6.4^{\circ}=$ $4.5 \%$ misses; $6.9^{\circ}=3.9 \%$ misses; $9.7^{\circ}=4.3 \%$ misses). The FA rate was $5.5 \%$.

In a replication of the one-target condition, we used set sizes of $1,25,35$, and 45 . Ten subjects searched for a red vertical target. Distractors were green vertical and red horizontal. In this replication, for set sizes greater than 1 $(25,35$, and 45 averaged), the slope of the eccentricity function was $39.1 \mathrm{msec} / \mathrm{deg}$, similar to the $32.8 \mathrm{msec} /$ deg eccentricity slope shown for this condition in Figure 3. With a set size of 1 , the eccentricity function had a slope of just $3.9 \mathrm{msec} / \mathrm{deg}$, shallower than the twotarget condition shown in Figure 5.

One might object that the subjects could readily foveate the single item, since the stimulus remained visible

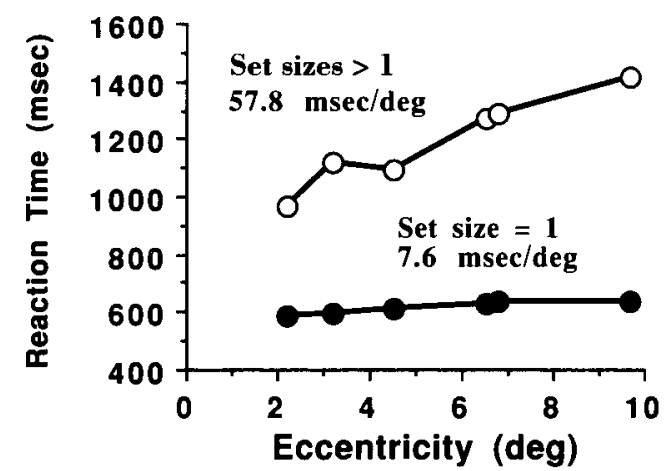

Figure 5. Results of Experiment 2 showing much less marked eccentricity effects when only a single item was presented.

until the subjects responded. As part of Experiment 4B, described more fully later, we repeated the Set Size 1 condition with briefly flashed stimuli $(180 \mathrm{msec})$. The target was a red vertical line, distractors were red horizontal and green vertical, and the slope of the eccentricity function was $4.6 \mathrm{msec} / \mathrm{deg}$, essentially the same as the $3.9 \mathrm{msec} / \mathrm{deg}$ slope reported for the same target continuously viewed. If eye movements had been important in this task, we would probably have seen a significant eccentricity effect reflecting the need to make eye movements to eccentric stimuli but not to the stimuli presented near fixation. In fact, it appears that this task with these stimuli produces very similar results with and without the possibility of eye movements.

The comparatively shallow eccentricity function obtained with a single item can be used to reject one hypothesis. It could be proposed that all items are processed simultaneously but that the time required to process any item in the periphery is much greater than the time required to process the same item in a more central location (cf. Carrasco \& Frieder, 1997). If that were the case, then a single item in the periphery should be found more slowly than should a single item near fixation. While we can reject this unlimited-capacity parallel processing model, we cannot reject the hypothesis that some limited processing capacity is divided among all items in the display. If more than 1 item was presented, less eccentric items would receive a larger share of the capacity. If that larger share produced faster RTs, then this sort of limitedcapacity parallel account could explain the results of Experiment 1 . Experiment 2 did not address this attentional hypothesis because, with only a single item, all processing resources could be directed to that item, and RTs would be fast for both central and peripheral targets.

Since there is some eccentricity effect even for a set size of 1 , it is tempting to propose that the eccentricity effect seen with larger set sizes is merely the Set Size 1 effect multiplied by the larger set size. That is, if it takes $50 \mathrm{msec}$ longer to examine a single item at $10^{\circ}$ eccentricity than at $2^{\circ}$, then one could argue that it would take $500 \mathrm{msec}$ longer to examine 10 items at $10^{\circ}$ eccentricity. However, this logic can explain the eccentricity effects in Experi- 
ment 1 only if we assume that central items (or groups of items) are scrutinized by attention before peripheral items (our proposed account of eccentricity effects). If the deployment of attention is random with regard to eccentricity, then the eccentricity effect for a set size of $N$ would not be $N$ times the effect for a set size of 1 . Consider, as an example, a display with 6 distractors, all presented at $5^{\circ}$. The target might be presented at $2^{\circ}$ or $10^{\circ}$. In either case, random search through the set ( 1 item at a time) will direct attention to an average of 3 of the $5^{\circ}$ distractors per trial. The contribution of the $5^{\circ}$ distractors will be the same in either case. The difference in RT would reflect the $50-\mathrm{msec}$ difference in the time required to process the $2^{\circ}$ versus the $10^{\circ}$ target item. This difference is independent of the number and position of distractors. A display of Set Size 1 would produce the same results as a display of set size $N$. If, however, $2^{\circ}$ items are examined before $5^{\circ}$ items, and if $5^{\circ}$ items are examined before $10^{\circ}$ items, then the RT for a target at $2^{\circ}$ will be significantly shorter than the RT for a $10^{\circ}$ target.

Suppose that processing is not by single items but by clumps of items. What happens to RTs if clumps of peripheral items are processed more slowly than clumps of central items? The answer is essentially the same as the answer for item-by-item processing. If clumps are processed in random order, then an average of half of the clumps will be processed prior to the processing of the clump containing a target. The time to process the set of "distractor clumps" will not be dependent on the eccentricity of the target clump. Any eccentricity effect will result only from differential processing of peripheral versus central target clumps.

To anticipate the argument of this paper, we believe that, in addition to any visual eccentricity effects, attention is being deployed centrally before being deployed peripherally. We will return to this model in the discussion of Experiment 4. For the present, we can conclude that eccentricity effects are not a simple product of slower processing of individual peripheral stimuli. Again, this is not to say that early vision factors do not influence visual search. However, the specific single-item factors tested in Experiment 2 are not adequate to explain the eccentricity effects in Experiment 1.

\section{EXPERIMENT 3 Effects of Crowding}

Reductions in sensitivity and acuity are not the only visual effects of increasing eccentricity. It is harder to identify an item if it is flanked by other items, and this "crowding" effect is more pronounced in the periphery than in the fovea (Levi et al., 1985; Toet \& Levi, 1992). Crowding could be another, low-level visual explanation of the eccentricity effects. Perhaps the slower RTs for eccentric targets reflect the additional effort required to confirm their identity in the presence of near neighbors. To test this hypothesis, we used a variation of the one-target condition of Experiment 1 using a set size of 4 items.

\section{Method}

Four items could be presented in a crowded display in which all items were in a single clump. Adjacent items had a center to center separation of $2.3^{\circ}$. The nearest contours of adjacent items were $1.1^{\circ}$ apart. This spacing was the same as the spacing in Experiment 1. The target, when present, was always bordered by the three other items. In the control condition, the four items (target +3 distractors) were placed randomly, resulting in much greater average separation between items in a very sparse display. The set size of 4 was used because 4 is the maximum possible number of flankers for a target presented in any of the corners of the display. Had we used the large set sizes of Experiment 1, we would have had nearby flanking distractors in the "uncrowded" condition, or had we forbade nearby flanking distractors, we would have provided a confounding cue to target location. The target would have been an unusually isolated item. Moreover, we assume that the prime lateral effects come from the nearest neighbors. Here, we have three near neighbors in the crowded condition.

Stimuli were flashed for $180 \mathrm{msec}$ in order to prevent voluntary eye movements. The subjects searched for a single, red vertical target. Ten subjects each were tested for 300 trials in both conditions.

\section{Results and Discussion}

As shown in Figure 6, the crowding manipulation had minimal impact on the eccentricity function. The difference between the slopes of the eccentricity functions was statistically significant ( $t$ test, $p<.05$ ). However, a substantial eccentricity effect was seen with the control stimuli, making it implausible that crowding effects were a substantial cause of the eccentricity effects of Experiment 1 . Though error rates rose somewhat with eccentricity, this was not a significant trend. Error rates were higher in the crowded condition $(7.8 \%)$ than in the control condition $(4.7 \%)[F(1,9)=5.53, p<.05]$. This difference was more marked for FAs than for misses. These FAs may represent "illusory conjunctions" (Treisman \& Schmidt, 1982) produced when the proximity of red horizontals and green verticals generates the illusory percept of a red vertical (Cohen \& Ivry, 1989). The eccentricity slopes were more shallow in this experiment than in the previous experiments because the set size was much smaller.

As with the effects of resolution on eccentricity that were examined in Experiment 2, we do not wish to argue that lateral inhibitory interactions such as crowding cannot contribute to eccentricity effects. Indeed, there is in-

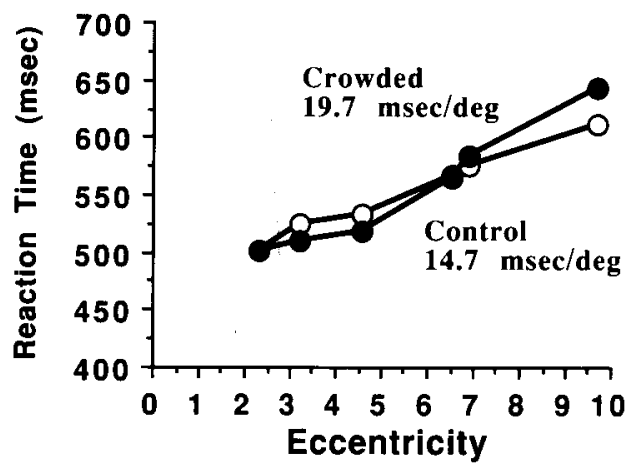

Figure 6. Results of Experiment 3 showing minimal effects of crowding on the eccentricity effect. 
teresting evidence for an attentional component to crowding (He, Cavanagh, \& Intriligator, 1996). We simply argue that substantial eccentricity effects remain in the control condition when crowding is minimized.

\section{EXPERIMENT 4A Cortical Magnification}

One way to compensate for the reduction in acuity and sensitivity in the periphery is to scale stimuli so that peripheral items are larger than central items. The relative amounts of cortical hardware devoted to processing different portions of the visual field can be mathematically described by a cortical magnification factor (Drasdo, 1991; Fiorentini \& Berardi, 1991; Levi et al., 1985; Rovamo \& Virsu, 1979; Wilson et al., 1990). If stimuli are scaled with the correct magnification factor, they will activate equivalent portions of cortex. Carrasco and Frieder (1997) report that "cortical magnification neutralizes the eccentricity effect" for their orientation feature search stimuli. In Experiment 4, we repeated their experiment using our color $X$ orientation conjunction search stimuli.

\section{Method}

There are various equations that have been used to estimate the appropriate magnification of peripheral stimuli. In Experiment 4, we used magnification $=1+E / E$, where $E$ is the eccentricity of the item, and $E^{\prime}$ is a scaling factor (Fiorentini \& Berardi, 1991). $E^{\prime}$ can take various values depending on the particular perceptual task. In Experiment $4, E^{\prime}$ was set to .7 , a conservative value intended to compensate not only for decline in acuity but also for the more severe decline in functions such as vernier acuity (Dennis Levi, personal communication, January 1996). The search for a red vertical target was rerun with scaled and unscaled stimuli. The scaled stimuli ranged in size from $0.7^{\circ} \times 0.2^{\circ}$ at the least eccentric loci to $2.3^{\circ}$ $\times 0.5^{\circ}$ at the most eccentric loci. The unscaled stimuli were $1.4^{\circ} \times$ $0.4^{\circ}$ at all loci. Set sizes were $1,10,20$, and 30 items. Set Size 1 data constituted a replication of Experiment 2. Distractors could appear in any of the 48 locations. Nine subjects were tested for 400 trials on each condition. In all other respects, the method and stimuli for Experiment 4A were the same as those used for Experiment 1.

In Experiment $4 \mathrm{~A}$, the subjects were asked to fixate while stimuli remained visible throughout the trial. In Experiment 4B, the stimuli were flashed to prevent eye movements.

\section{Results}

Figure 7 shows results averaged across all set sizes other than Set Size 1. Cortical magnification of the stimuli did not eliminate the eccentricity effect. Slopes of the eccentricity function were not significantly different $[t(8)=0.74, p>.4]$. If anything, search was somewhat slower for the scaled stimuli than for the unscaled. This could reflect the deleterious effects of using stimuli that are heterogeneous in size. Distractor heterogeneity is known to impede search (Duncan \& Humphreys, 1989).

Error rates increased modestly with set size and eccentricity, and they were somewhat greater for the scaled condition than for the control, as shown in Tables 1 and 2.

In Experiment $4 \mathrm{~A}$, the subjects were asked to fixate. However, eye movements were not monitored, and we cannot be sure that they did fixate. Thus, it is possible

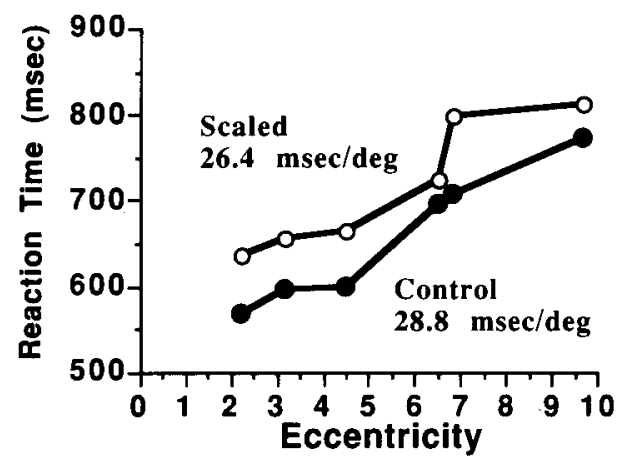

Figure 7. Results for Experiment 4A. Correcting for cortical magnification had a fairly minimal impact on the eccentricity effect in visual search with these continuously viewed stimuli.

that the effects of scaling the stimuli could have been obscured by eye movements. Accordingly, we repeated the scaling experiment with brief presentations intended to eliminate the effects of voluntary eye movements (cf. Carrasco \& Frieder, 1997).

\section{EXPERIMENT 4B Scaled Stimuli, Briefly Presented}

\section{Method}

The basic design of Experiment 4B was similar to that of Experiment 4A. Eight subjects were tested. Rather than remaining visible until the subject responded, the search stimulus was flashed for $180 \mathrm{msec}$ on each trial. The stimuli were not masked, since our only interest was in preventing voluntary refixations. Set sizes were 1,8 , 10 , and 12 items. The smaller set sizes were used in order to keep error rates within reasonable bounds. Experiment $4 \mathrm{~B}$ was otherwise similar to the previous experiments.

\section{Results}

Turning first to the data for Set Size 1, as shown in Figure 8, scaling did eliminate the linear increase in RT

Table 1

Error Rates as a Function of Set Size

\begin{tabular}{cccccc}
\hline & \multicolumn{2}{c}{ Misses } & & \multicolumn{2}{c}{ False Alarms } \\
\cline { 2 - 3 } \cline { 5 - 6 } Set Size & Scaled & Control & & Scaled & Control \\
\hline 1 & 0.3 & 1.1 & & 1.5 & 2.7 \\
10 & 1.8 & 0.9 & & 1.9 & 2.5 \\
20 & 2.3 & 0.8 & & 3.2 & 1.7 \\
30 & 3.3 & 1.1 & & 1.8 & 0.9 \\
\hline
\end{tabular}

Note-All values are expressed in percentages.

Table 2

Error Rates as a Function of Eccentricity

\begin{tabular}{ccc}
\hline & \multicolumn{2}{c}{ Misses } \\
\cline { 2 - 3 } Eccentricity & Scaled & Control \\
\hline $2.3^{\circ}$ & 1.9 & 0.2 \\
$3.2^{\circ}$ & 1.4 & 0.9 \\
$4.6^{\circ}$ & 0.7 & 0.2 \\
$6.5^{\circ}$ & 0.7 & 1.1 \\
$6.9^{\circ}$ & 3.7 & 1.8 \\
$9.7^{\circ}$ & 3.2 & 1.4 \\
\hline
\end{tabular}

Note-All values are expressed in percentages. 
with eccentricity. The U-shaped form of the data suggests that the smallest items may have been small enough to have been put at a comparative disadvantage by the scaling. Figure 9 shows the results for Set Sizes 8, 10, and 12. In this experiment, the eccentricity slope was significantly reduced in amplitude in the RT data of the scaled condition $[t(7)=4.5, p<.005]$ as well as in the error data (see Table 3).

However, scaling did not eliminate the eccentricity function. Eccentricity slopes were still significantly greater than zero $[t(7)=4.1, p<.005]$. Moreover, error rates were higher in the scaled condition than in the control condition at every set size (Table 4).

\section{Discussion}

Experiment 4A showed little or no effect of scaling. Experiment 4B showed some effect. Carrasco and Frieder (1997) found that scaling their stimuli all but eliminated the eccentricity effect. What are we to make of this range of results? The variety of results probably illustrates the interaction of attentional and visual factors in the eccentricity effect. Experiment 4B can be seen as a partial replication of Carrasco and Frieder. They used much smaller stimuli in their unscaled condition $\left(0.1^{\circ} \times 0.5^{\circ}\right)$ than we used in our control condition $\left(0.4^{\circ} \times 1.4^{\circ}\right)$. As a result, their magnified peripheral stimuli were much more identifiable when magnified. This can be seen in the decline in error rate at their highest eccentricity from $34 \%$ with the unscaled stimuli to about $15 \%$ with the larger scaled stimuli. In Experiments $4 \mathrm{~A}$ and $4 \mathrm{~B}$, our unscaled stimuli were intermediate in size between the largest peripheral and the smallest central stimuli in the scaled condition. When these stimuli were briefly flashed in Experiment 4B, the smaller central stimuli in the scaled condition were harder to detect than the larger central stimuli in the control condition. This is seen in the elevated RTs and errors at low eccentricities for the scaled condition in Experiment $4 \mathrm{~B}$. When the stimuli were visible for a longer duration in Experiment 4A, all stimuli at all eccentricities seem to have been far enough above threshold that the scaling manipulation had minimal effect.

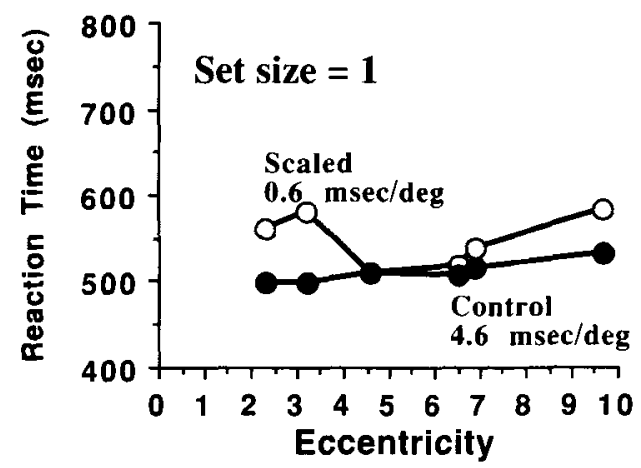

Figure 8. Effects of scaling for cortical magnification on speed of identification of a single item.

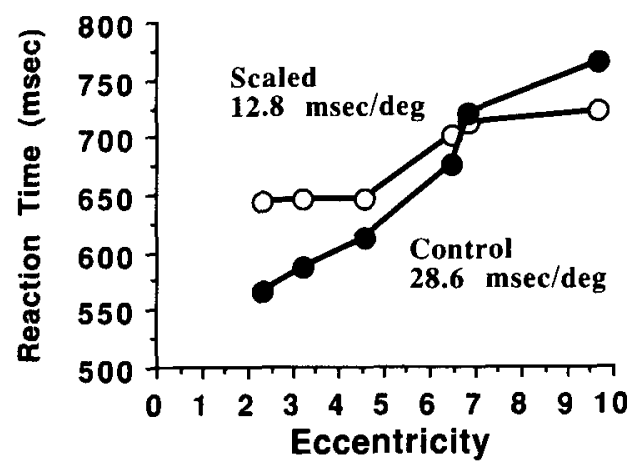

Figure 9. Effects of scaling for cortical magnification on speed of visual search.

While eye movements were possible in Experiment 4A, their predicted effect would be to reduce the eccentricity effect (by averaging effective eccentricity). The eccentricity effect was larger in the scaled condition of Experiment 4A than in that of Experiment 4B. Moreover, Carrasco has argued that eye movements do not account for the eccentricity effect (Carrasco et al., 1995; Carrasco \& Frieder, 1997). A parsimonious account of the data on scaling effects would be that changing the size of the stimulus has an effect on visual search when that size change significantly alters the subject's sensitivity to the stimulus. Indeed, it should be possible to reverse the eccentricity effect if one used large enough peripheral stimuli and small enough central stimuli. If, however, all stimuli are far enough about threshold for sensitivity considerations to be moot, then scaling has little impact. We suggest that the cortical magnification factor has no special status here. We hypothesize that, by making peripheral stimuli larger, we are merely making it possible for them to attract attention and to overcome the normal penalty that peripheral stimuli have relative to the central stimuli.

\section{AN ATTENTIONAL ACCOUNT OF ECCENTRICITY EFFECTS}

We suggest that visual factors such as cortical magnification and crowding do not produce the eccentricity effects in search. Rather, we suggest that visual factors modulate the deployment of attention. The result is that, all else being equal, attention is deployed to central stimuli before peripheral stimuli. This account assumes serial deployment of attention from item to item. Alternatively, one could assume that processing capacity is directed to all items at once but that this capacity is limited. The role of attention would be to allocate capacity to different items. Models of this sort can produce the same general patterns of RTs as serial models (Kinchla, 1974; Mordkoff \& Egeth, 1993; Palmer, 1995; Ratcliff, 1978; Townsend, 1971, 1976, 1990). In a limited-capacity version of an attentional account of eccentricity effects, more capacity would be allocated to central items than to 
Table 3

Errors Rates as a Function of Eccentricity for Experiment $4 B$

\begin{tabular}{ccc}
\hline & \multicolumn{2}{c}{ Misses } \\
\cline { 3 - 3 } Eccentricity & Scaled & Control \\
\hline $2.3^{\circ}$ & 9.0 & 3.0 \\
$3.2^{\circ}$ & 9.0 & 2.0 \\
$4.6^{\circ}$ & 7.0 & 3.0 \\
$6.5^{\circ}$ & 12.0 & 9.0 \\
$6.9^{\circ}$ & 8.0 & 9.0 \\
$9.7^{\circ}$ & 6.0 & 14.0 \\
\hline
\end{tabular}

Note-All values are expressed in percentages.

peripheral items. Both serial and limited-capacity parallel accounts are described below.

\section{A SERIAL MODEL OF ECCENTRICITY EFFECTS}

In serial models of visual search, attention is presumed to be deployed from item to item until a target is found or until search is terminated without a target being found (e.g., Treisman \& Gelade, 1980). Conjunction searches, such as those used here, produce results that are inconsistent with serial deployment of attention to each and every item because the slopes of RT $\times$ set size functions are too shallow (e.g., Nakayama \& Silverman, 1986; Treisman \& Sato, 1990; Wolfe et al., 1989; Zohary \& Hochstein, 1989). In a serial self-terminating search, subjects will need to examine, on average, one half of the presented items on trials when a target is present. Thus, one estimate of the speed of attentional deployment can be obtained by doubling the slope of the RT $\times$ set size function for target-present trials. Computed in this manner, data from conjunction searches yield estimates of an attentional redeployment every $10-20 \mathrm{msec}$. However, other work shows that attention redeploys no faster than once ever 40-60 msec (Sagi \& Julesz, 1985) and, perhaps, much slower than that (Duncan, Ward, \& Shapiro, 1994). The Guided Search model proposed a solution. In cases where some basic feature information was available, preattentive information could "guide" the deployment of attention so that attention would not need to be deployed at random from item to item. For instance, if the target were green and vertical, parallel color processes could guide attention toward green items, while parallel orientation processes guided attention toward vertical items. In the model, guidance takes the form of "activation" of the appropriate items. The various sources of guidance/activation are summed in an "activation map." Attention is deployed first to the item with the most activation. Thereafter, items are examined in decreasing order of activation-a ranking of the preattentive likelihood that they are targets (Wolfe, 1994; Wolfe et al., 1989; Wolfe \& Gancarz, 1996). Recent modifications of feature integration theory include similar mechanisms (Treisman, 1993).

In the third generation of the Guided Search model (GS3; Wolfe \& Gancarz, 1996), a small modification of the guided search architecture produces eccentricity effects. Specifically, the "retinal" image is distorted by a $\log$ polar transform that mimics the cortical magnification factor (Schwartz, 1977), overrepresenting the fovea and underrepresenting the periphery. This, in turn, produces greater attention-guiding activation for an item presented at the fovea than for an equivalent item presented in the periphery. In effect, this allows the effects of cortical magnification (Carrasco \& Frieder, 1997) to be reflected in the guidance of attention.

\section{A LIMITED-CAPACITY PARALLEL MODEL}

The central concept of guided search can be easily adapted to limited-capacity parallel models of visual search (Wolfe, 1994). In a limited-capacity parallel version of guided search, preattentive processes would regulate the allocation of attentional resources rather than the deployment of some attentional "spotlight." To account for eccentricity effects, one merely needs to assume that, all else being equal, attentional resources are preferentially allocated to the central portions of the field. If less of a limited attentional resource is available to a peripheral target, RT should be slowed just as it is slowed when the resource must be spread over many items in a large set size display. The account of Carrasco and Frieder (1997) combines aspects of the serial and parallel models described here.

In the rest of this paper, we will discuss an account of the eccentricity effect that assumes serial deployment of attention from item to item. In all cases, a limited-capacity parallel account can be created by substituting a biased allocation of a limited attentional resource for a biased deployment of attention.

\section{EXPERIMENT 5 \\ Searches With All Items on a Trial at a Single Eccentricity}

It is possible to design a search task for which visual and attentional accounts of the eccentricity effect make qualitatively different predictions. Suppose that all items are at the same eccentricity. A visual account of eccentricity effects predicts that the RT should depend on that eccentricity. If searches were performed with rings of items at one eccentricity on some trials and another eccentricity on other trials, then the average RTs, plotted as a function of the eccentricity of the ring, should show a standard eccentricity effect. If, on the other hand, eccen-

Table 4

Error Rates as a Function of Set Size for Experiment $4 B$

\begin{tabular}{ccc}
\hline Set Size & Scaled & Not Scaled \\
\hline 1 & 2.7 & 1.2 \\
8 & 7.4 & 5.0 \\
10 & 9.7 & 7.3 \\
12 & 15.1 & 9.6 \\
\hline
\end{tabular}

Note-All values are expressed in percentages. 
tricity effects are the by-product of a bias toward searching central items before peripheral items, then the eccentricity of the ring of items should make no difference as long as the items can be seen adequately at all eccentricities. In rings of items at a single eccentricity, a target item has the same chance of being visited by attention first when the ring eccentricity is large as it does when ring eccentricity is small. In other words, the visual hypothesis predicts effects of absolute eccentricity, whereas the attentional hypothesis predicts effects of relative eccentricity. These predictions were tested in Experiment 5.

\section{Method}

The subjects in Experiment 5 were tested in a $2 \times 2$ array of conditions: All stimuli in a single trial could be presented at equal eccentricity, or they could be presented at random eccentricities (as in the previous experiments). For each of those types of display, stimuli could be scaled in accord with the cortical magnification experiment (as in Experiment 4), or they could be unscaled, all items a single size (as in the control conditions of Experiment 4). Scaled conditions were run in order to obtain more data on the role of the cortical magnification factor.

In each of these four conditions, targets could appear at one of four eccentricities $1.8^{\circ}, 3.5^{\circ}, 5.3^{\circ}$, and $7.0^{\circ}$ from fixation. Rather than 49 possible stimulus locations in a $7 \times 7$ array, stimuli could appear in 32 locations of 4 concentric rings with 8 positions per ring. Any item in a display could be a target. On each trial, the set size was 4 or 8 items, 8 items being the maximum number that could be placed at the same ecentricity in this display. In this display, stimulus density decreases as eccentricity increases. Given the small effects of lateral interactions with similar stimuli in Experiment 3, these density variations seem unlikely to have a major effect on the results.

The four conditions of Experiment 5 were run in separate blocks of 400 trials. Order of testing was pseudorandom across the 10 subjects in the experiment. Other aspects of the stimuli and the experimental design followed those of the previous experiments.

\section{Results}

Figure 10 shows the eccentricity functions for the four conditions. Looking first at the equal eccentricity conditions, eccentricity effects were small for the unscaled stimuli and were absent for the scaled stimuli. Thus, when targets and distractors were at the same eccenticity within a trial, the RT for a target at $7^{\circ}$ was about the same as the RT for a target at $1.8^{\circ}$. No particular difficulty was encountered when search was occurring in the periphery unless there were distractors lying closer to the fovea than to the target. Error rates were unremarkable (Table 5).

As in the previous experiments, the effect of these less eccentric items can be seen in the random eccentricity conditions of Experiment 5. These essentially replicated the results of Experiment 4 . There was a substantial slope to the eccentricity function for unscaled stimuli. This is the standard eccentricity effect. In another partial replication of Carrasco and Frieder (1997), the slope of the eccentricity function was significantly reduced by scaling the stimuli $[F(1,9)=15.3, p<.004]$. Scaling reduced but did not eliminate the eccentricity effect. Overall, RTs for the scaled condition were longer than those for the unscaled condition, consistent with the finding that
Table 5

Error Rates as a Function of Eccentricity for Experiment 5

\begin{tabular}{|c|c|c|c|c|}
\hline \multirow[b]{2}{*}{ Eccentricity } & \multicolumn{2}{|c|}{ Equal Eccentricity } & \multicolumn{2}{|c|}{ Random Eccentricity } \\
\hline & Scaled & Unscaled & Scaled & Unscaled \\
\hline $1.8^{\circ}$ & 3.4 & 4.8 & 3.5 & 1.5 \\
\hline $3.5^{\circ}$ & 2.4 & 3.8 & 1.7 & 1.9 \\
\hline $5.3^{\circ}$ & 3.6 & 3.8 & 4.4 & 4.0 \\
\hline $7.0^{\circ}$ & 4.0 & 3.0 & 5.6 & 4.4 \\
\hline
\end{tabular}

Note-All values are expressed in percentages.

heterogeneous distractors slow search (Duncan \& Humphreys, 1989).

As one way to capture the difference between visual and attentional accounts of the eccentricity, consider two cases where the target was at $7^{\circ}$ eccentricity. In the equal eccentricity conditions, all distractors were also at $7^{\circ}$. In the random eccentricity conditions, some of those distractors were moved closer to the fovea. This should have improved the visual representation by decreasing the average eccentricity of the stimuli. However, it made the search task harder. This is difficult to explain visually; but it makes sense attentionally. The less eccentric distractors were more likely to attract attention.

Eccentricity functions and set size functions. Carrasco et al. (1995) raised the concern that $\mathrm{RT} \times$ set size functions might be modulated by eccentricity effects. The attentional account of eccentricity effects predicts that the set size and eccentricity effects should covary when task difficulty is varied while the spatial distribution of stimuli is held constant. Take the case where stimuli are randomly distributed in the display, and consider two tasks: a relatively easy search task such as the conjunction search used here and a harder "serial" task such as a search for a 2 among $5 \mathrm{~s}$. The harder task will require attention to be deployed to more distractors, and, as a result, the RT $\times$ set size slope will increase and the eccentricity slope will increase as attention is deployed to a greater proportion of the distractors lying between the fovea and the target. This issue was addressed in Experiment 6 .

While set size and eccentricity effects should covary as task difficulty varies, the two effects should be independent when task difficulty is held constant while the spatial layout of the stimuli is changed. Specifically, Figure 10 shows a large difference in eccentricity functions between equal eccentricity and random eccentricity conditions. According to the attentional account, eccentricity effects depend on the number of distractors lying between the target and the fovea. The number of more central distractors varied with target eccentricity in the random eccentricity condition but not in the equal eccentricity condition. Thus, the random condition produced the larger eccentricity effects. By contrast, the set size effect did not depend on this variable as long as targets were presented equally at all eccentricities. The random versus equal eccentricity manipulation should not have influenced RT $\times$ set size functions 

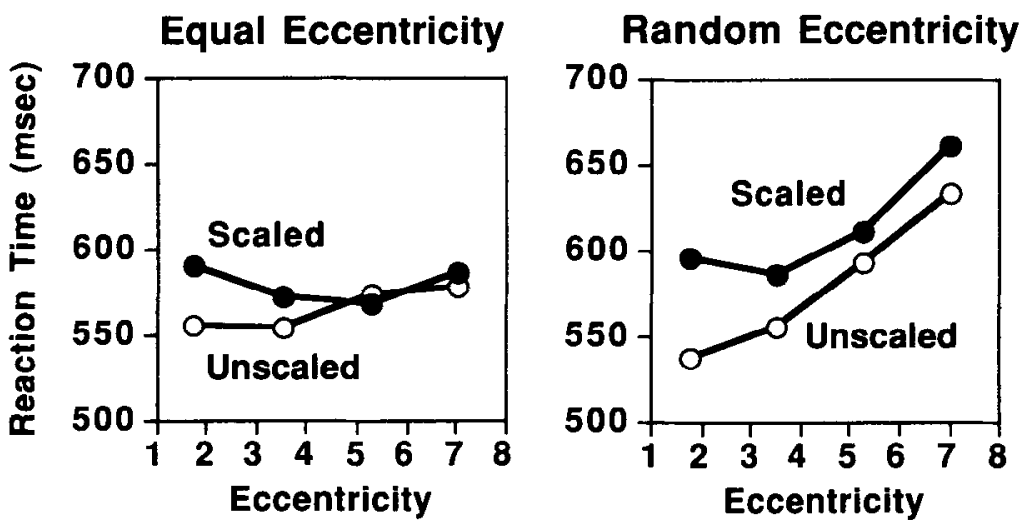

Figure 10. Eccentricity functions for Experiment 5. Note that equal eccentricity conditions produced minimal eccentricity functions.

except, perhaps, in the random eccentricity-scaled condition where distractor heterogenity may have made the task harder (Duncan \& Humphreys, 1989).

Figure 11 shows the $\mathrm{RT} \times$ set size functions for the four conditions of Experiment 5. In both of the equal eccentricity conditions and in the random eccentricity-unscaled condition, all items were the same size on any given trial. Note that the slopes of the RT $\times$ set size functions were very similar for these three conditions. Manipulations that alter the eccentricity effect can leave RT $X$ set size effects unchanged. In the fourth condition, random eccentricity-scaled items were heterogeneous in size. The RTs in this condition were slower, and the RT $X$ set size function was steeper. Tukey post hoc comparisons $(p<.05)$ show that the equal eccentricity-scaled, equal eccentricity-unscaled, and random eccentricityunscaled slopes were not significantly different from each other. The random eccentricity-scaled slopes were significantly steeper than the random eccentricity-unscaled slopes but were not different from the equal eccentricityscaled and equal eccentricity-unscaled slopes.

Looking more closely at the equal eccentricity conditions, we can ask whether different R'T $\times$ set size functions were obtained for rings of items at different eccentricities. Was the slope of the RT $\times$ set size function greater for a ring of items at a more eccentric location? The answer is no. Set size slopes were not significantly altered by eccentricity $[F(3,27)=0.303$, n.s.].

\section{Discussion}

An attentional model that sees the eccentricity effect as a product of a central bias in the allocation of attention fares quite well in this experiment. These models predict that placing distractors at the same eccentricity as the target should largely eliminate the eccentricity effect. The data followed this prediction. RTs pooled over several equal eccentricity displays show small or absent eccentricity effects. Attentional allocation models also predict that placing distractors at the same eccentricity would have no effect on the RT $\times$ set size functions. That prediction was also substantially borne out. It would be harder to explain the results of Experiment 5 without invoking attention.

Relationship of eccentricity and set size functions. The results of Experiment 5 shed light on the relationship between RT $\times$ set size functions and eccentricity functions. As we found in Experiment 6 (below), set size and eccentricity functions should covary when task difficulty is changed. For both measures, harder tasks require more attention to distractors. The results of Exper-

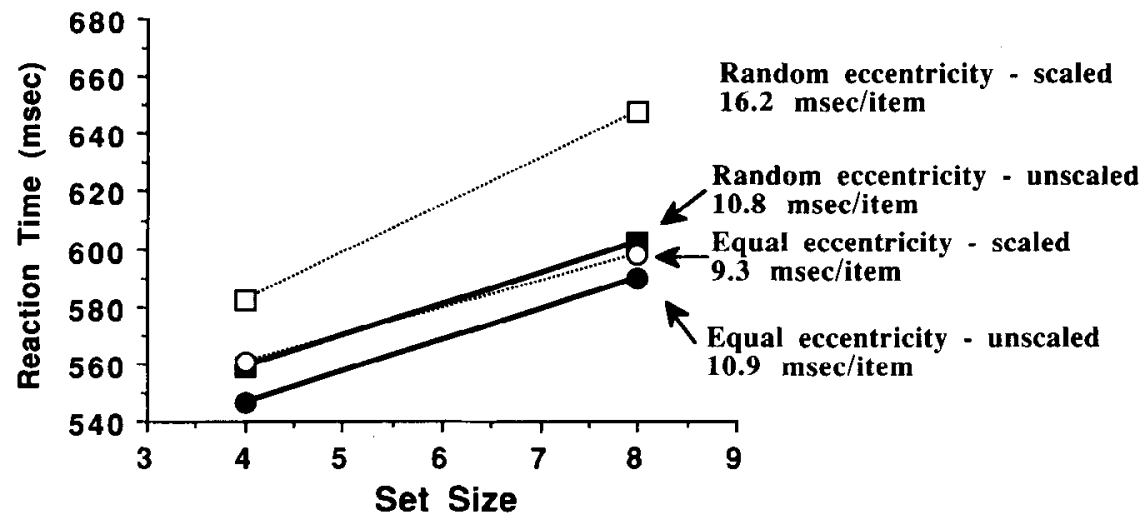

Figure 11. RT $\times$ set size functions for the four conditions of Experiment 5 . 
iment 5 show that set size functions can be independent of changes in the spatial distribution of distractors, whereas eccentricity functions are dependent on that distribution. Thus, we would argue, eccentricity effects and set size effects are separate effects that can be influenced by the same variables. Set size effects are not an artifact of eccentricity effects.

Carrasco, however, makes an important point when she argues that eccentricity needs to be considered in the design of visual search experiments. To use an extreme example, if targets were always presented at fixation, the presence of distractors at more peripheral locations will have much less effect than if the targets were presented in the periphery. The RT $\times$ set size effect would all but vanish for targets presented at fixation (see Experiment 6). We have simulated results for four less far-fetched experimental conditions similar to the stimulus configurations used in the experiments reported here.

Condition 1: A $7 \times 7$ array of stimuli with targets presented randomly in any location.

Condition 2: A $7 \times 7$ array of stimuli with targets restricted to 24 of the 49 locations on eight radial arms as shown in Figure 1.

Condition 3: An array of 7 concentric rings of 7 items with target presented randomly.

(For Conditions 1-3, we simulated a completely serial search with search proceeding from item to item in strict order of eccentricity. Thus, if the target was at eccentricity 2.5 , attention would be deployed to all distractors with eccentricities less than 2.5 and to none of the distractors with eccentricities greater than 2.5 . If a distractor had an eccentricity identical to the target, it had a $50 \%$ chance of being attended on that trial.)
Condition 4: A $7 \times 7$ array as in Condition 1; however, we assumed no eccentricity function and simply allowed attention to be deployed randomly in a serial self-terminating manner.

The simulation was run for 700 trials -100 trials at each of seven set sizes from 5 to 35 items. No actual stimuli were involved. The simulation simply generated a list of items in accordance with the rules for target and distractor placement and then determined how many distactors would be checked on that trial before the target was checked. The simulation did not make "errors." Results are shown in Figure 12.

The RT units in Figure 12 are attentional deployments: How many distractors were attended before the target was found? Condition 4 is a classic serial self-terminating search. RT in Condition 4 should and does approximate $0.5 \times(\mathrm{SS}-1)$, where SS is the set size. Conditions 1 and 3 produce essentially the same result. As long as target location is chosen randomly, the subject will need to attend to half of the distractors on average. However, in Condition 2, targets are constrained to be equally probable at all eccentricities, whereas distractors are not similarly constrained. In this case, the average distractor location is more peripheral than the average target location. As a result, the slope of the RT $\times$ set size function is about $20 \%$ shallower in Condition 2 . The bottom line is that RTs and RT $\times$ set size functions can be altered if the constraints on target location are not the same as the constraints on distractor location. These effects are a byproduct of eccentricity effects, and so, in that sense, set size and eccentricity effects interact. However, the results of Experiment 5 show that the two effects can be independent of each other. Conditions 1 and 4 of the model make the same point. The RT $\times$ set size functions, produced by Conditions 1 and 4 , are the same even though

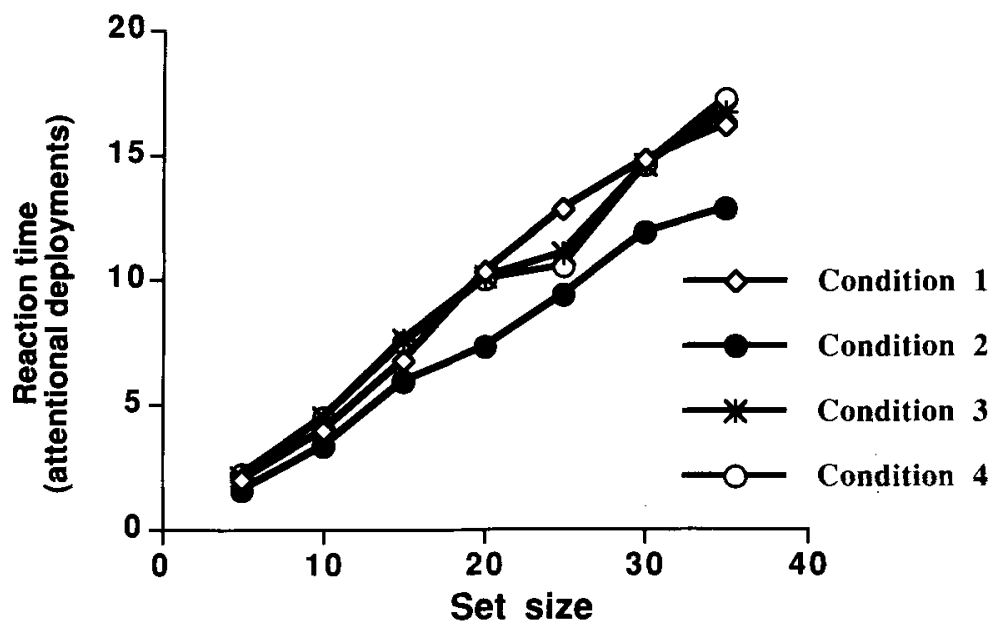

Figure 12. Results of a simulation of the effect of different stimulus configurations on $\mathrm{RT} \times$ set size functions. Results for condition 2 differed from all results for other conditions. See text for details. 
Condition 1 assumes a strong eccentricity effect, whereas Condition 4 assumes none.

\section{EXPERIMENT 6 \\ Interactions of Eccentricity, Set Size, and Task Difficulty}

The attention allocation model of the eccentricity effect makes two predictions that are tested in the final experiment.

Prediction 1: Set size functions should vary with eccentricity. If search proceeds roughly from center to periphery, it follows that the effective set size for a centrally located target is smaller than the effective set size for a peripherally located target. To find a target located near the center of the display, the observer will search through only those few items near the center. The observer will need to search much more widely in order to find a peripheral target. This means that RT $\times$ set size effects should be larger for peripheral targets than for central targets.

Prediction 2: Harder tasks that produce steeper RT $\times$ set size functions should produce steeper eccentricity functions. The logic is similar to that of Prediction 1. The impact of increasing the effective number of distractors will be greater for harder tasks than for easier tasks. Increasing set size increases the number of distractors. Increasing target eccentricity increases the effective number of distractors. Therefore, if set size functions are steeper, eccenticity functions should be steeper.

Note that there are also visual accounts of these effects (e.g., Carrasco \& Frieder, 1997). The purpose of Experiment 6 was less to distinguish between visual and attentional accounts than to further explore the relationship between eccentricity and set size.

\section{Method}

In principle, we could examine the data from any of the previous experiments to obtain an estimate of the slope of the RT $\times$ set size function for a target presented at a specific location in the display. However, the number of trials going into any such estimate would be very small. Accordingly, in Experiment 6,18 subjects were subjected to 1,500 trials in each of two tasks. In the conjunction task, the subjects searched for a red vertical target among green vertical and red horizontal distractors. Items were $1.1^{\circ} \times 0.3^{\circ}$. In the serial search task, the subjects searched for a 2 among 5 (a 2 among $5 \mathrm{~s}$, or a mirror-reversed $\mathrm{S}$ among Ss). Items were $1.5^{\circ} \times 0.75^{\circ}$. Targets were presented on $67 \%$ of the trials. Set sizes were $4,16,28$, and 40 items for the conjunction task, and $4,10,16$, and 22 items for the 2 -among-5s task. Smaller set sizes were used in the 2-among-5s task when pilot data suggested that error rates were unacceptably high for the largest set sizes. Targets could be presented in any of the 24 locations shown in Figure 1. In all other respects, the methods were similar to those in the previous experiments.

\section{Results and Discussion}

The slopes of the RT $\times$ set size functions in the serial search condition were $30 \mathrm{msec} /$ item on target-present trials and $89.7 \mathrm{msec} /$ item on target-absent trials. This is consistent with a serial self-terminating search, albeit with rather conservative search termination on blank trials (Chun \& Wolfe, 1996). The slopes in the conjunction search condition were $4.4 \mathrm{msec} /$ item on target-present trials and $21.0 \mathrm{msec} /$ item on target-absent trials. This is consistent with an efficient, guided search, again with conservative search termination on blank trials.

Figure 13 shows the eccentricity functions for the two conditions. Table 6 gives error rates as a function of set size and eccentricity for the two conditions. In Figure 13, each data point represents the average of the median RTs at one location. Thus, there are four data points for each eccentricity. The conjunction task produced a markedly shallower eccentricity function than did the 2-among-5s

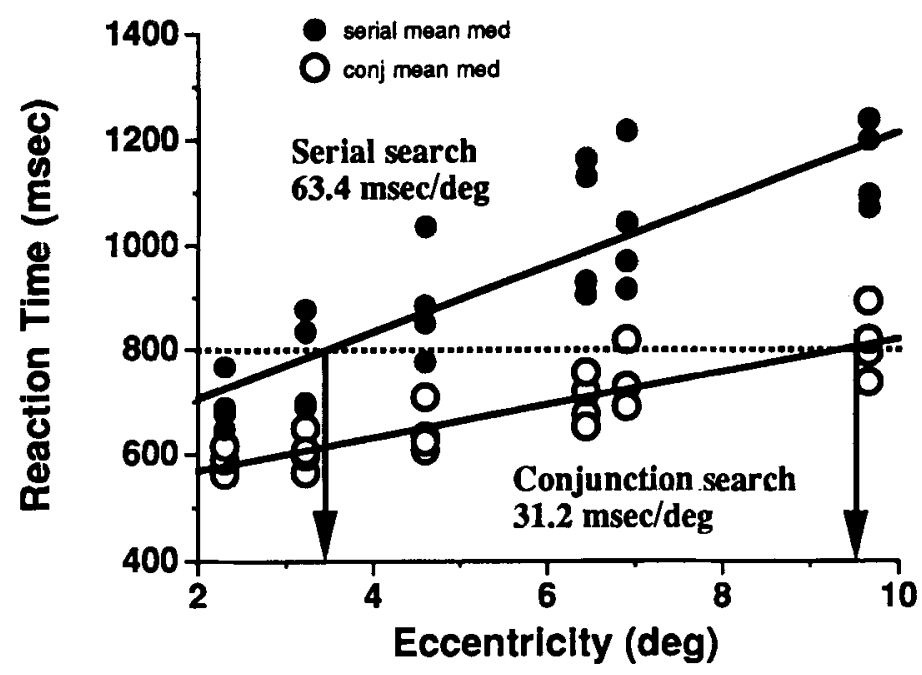

Figure 13. RT as a function of target eccentricity for Experiment 6. Dotted line shows an 800-msec threshold, and the arrows show the resulting UFOV estimates for the two search tasks. 
Table 6

Error Rates as a Function of Set Size and Eccentricity for Experiment 6

\begin{tabular}{|c|c|c|c|c|c|c|c|c|}
\hline \multirow[b]{3}{*}{ Eccentricity } & \multicolumn{4}{|c|}{ Conjunction Task } & \multicolumn{4}{|c|}{ 2-Among-5s Task } \\
\hline & \multicolumn{4}{|c|}{ Set Size } & \multicolumn{4}{|c|}{ Set Size } \\
\hline & 4 & 16 & 28 & 40 & 4 & 10 & 16 & 22 \\
\hline \multicolumn{9}{|c|}{ Misses } \\
\hline $2.3^{\circ}$ & 0.3 & 0.3 & 0.3 & 1.4 & 1.1 & 0.8 & 2.3 & 5.4 \\
\hline $3.2^{\circ}$ & 0.7 & 1.0 & 0 & 0.6 & 0.5 & 0.4 & 2.9 & 6.0 \\
\hline $4.6^{\circ}$ & 0.0 & 0.4 & 1.2 & 1. & 0.4 & 1.8 & 4.0 & 8.3 \\
\hline $6.5^{\circ}$ & 1.4 & 1.0 & 0.8 & 1.9 & 1.0 & 1.4 & 4.1 & 7.6 \\
\hline $6.9^{\circ}$ & 1.3 & 1.3 & 2.0 & 2.1 & 0.8 & 1.3 & 4.3 & 10.2 \\
\hline $9.7^{\circ}$ & 1.6 & 2.4 & 1.5 & 1.4 & 2.7 & 2.8 & 3.8 & 5.7 \\
\hline \multicolumn{9}{|c|}{ False Alarms } \\
\hline $2.3^{\circ}$ & 2.0 & 2.1 & 1.0 & 0.0 & 0.0 & 1.1 & 2.6 & 2.2 \\
\hline $3.2^{\circ}$ & 2.7 & 3.3 & 0.5 & 1.1 & 2.5 & 1.9 & 3.0 & 1. \\
\hline $4.6^{\circ}$ & 2.2 & 0.5 & 0.0 & 0.0 & 1.4 & 2.4 & 1.6 & 2.5 \\
\hline $6.5^{\circ}$ & 1.1 & 0.5 & 0.6 & 2.3 & 1.0 & 2.0 & 2.2 & 2.1 \\
\hline $6.9^{\circ}$ & 2.6 & 1.1 & 1.4 & 0.0 & 2.2 & 1.6 & 3.5 & 0.5 \\
\hline $9.7^{\circ}$ & 1.2 & 1.2 & 0.5 & 0.5 & 0.5 & 2.5 & 2.7 & 3,1 \\
\hline
\end{tabular}

Note-All values are expressed in percentages.

task. This makes sense in the context of the model outlined earlier. The bias toward central locations was one source of guidance in the deployment or allocation of attention. In the serial task, it was the only source of guidance. In the conjunction task, color and orientation provided guidance of their own, diluting the effects of eccentricity and reducing the eccentricity slope. Errors increased with both set size and eccentricity, replicating the pattern reported by Carrasco et al. (1995).

As noted in the introduction, using very different tasks, eccentricity effects have been used to support the notion of a UFOV (Ball et al., 1988; Sanders, 1970, 1993). The results shown in Figure 13 show that it would be unwise to think of a UFOV as a fixed attentional aperture used by a subject to look at the world. The size of the UFOV is going to be task-dependent. For instance, we could define a UFOV as the portion of the field in which our search task could be successfully completed in $800 \mathrm{msec}$. Looking at Figure 13, we can see that this UFOV would have a radius of about $3^{\circ}$ as assessed by the serial task and $10^{\circ}$ as assessed by this conjunction task.

Turning to the interaction of the set size slopes with eccentricity, the data support the first prediction set forth above. Figure 14 shows the average slope of the RT $\times$ set size function computed at each eccentricity. The RT $\times$ set size slopes were shallower for targets near fixation than they were for more eccentric targets. This would be predicted if subjects preferentially searched near fixation first. In effect, the relevant set size increased as the eccentricity of the target increased. The effect was much smaller for the conjunction task because guidance by color and orientation made the overall search task much more efficient than the 2-among-5s search task.

While the data presented here support the idea that attention is deployed or allocated to central items before peripheral items, the results from Experiment 6 can also be used to reject the strictest and simplest version of that model. Several predictions can be made if it is proposed that attention is deployed in strict order of eccentricity. First, consider the relationship shown in Figure 14. The number of possible item locations increased with the square of the eccentricity when items were presented randomly in a uniform grid of possible locations. If search was a strict function of eccentricity, then it follows that the effective set size and, thus, the slopes of the RT $\times$ set size functions should increase with the square of eccentricity. However, the data in Figure 14 show a decelerating function for both serial and conjunction searches. Moreover, the slopes at the smallest eccentricity were excessively steep. The RT $\times$ set size functions from blank trials (where every item must be examined if search is serial and exhaustive) can be used to predict the targetpresent slope for a given eccentricity. For instance, at the smallest eccentricity, there are only four locations. In a serial self-terminating search, only two of these locations will be examined on an average trial. The resulting slope should be $2 / 48$ of the blank trial slope or $3.7 \mathrm{msec}$ in this case. This estimate is substantially less than the observed $11.5 \mathrm{msec} / \mathrm{item}$. The combination of a decelerating function and the overly large slopes at small eccentricities suggests that the deployment of attention toward the center is a noisy process - a bias and not a strict rank ordering. Recall from the earlier simulation that the exact nature of the underlying eccentricity function should not influence RT $\times$ set size if target and distractor locations are sampled in an equivalent manner.

A second set of predictions concerns the relationship between set size slopes and eccentricity slopes. Both of these depend in a linear manner on the speed with which subjects can process individual items and on the total number of relevant items in the display. It follows that the two slopes should be related to each other. A subject with a shallow set size slope should have a shallow eccentricity function. This was the case, as is shown in Figure 15.

There is a respectable correlation between set size slopes and eccentricity functions for each task. Figure 15 also makes it clear that the relationship between set size slopes and eccentricity functions was different for the two tasks. This was probably related to the differences between the stimuli in the two conditions, but further re-

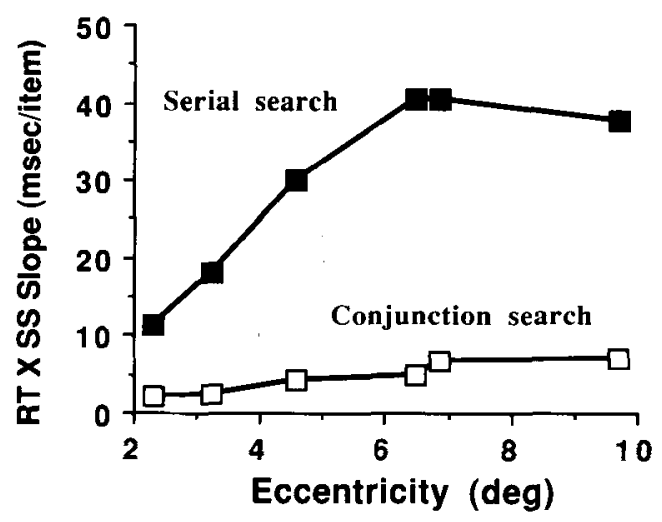

Figure 14. RT $\times$ set size slopes as a function of eccentricity for Experiment 6. 


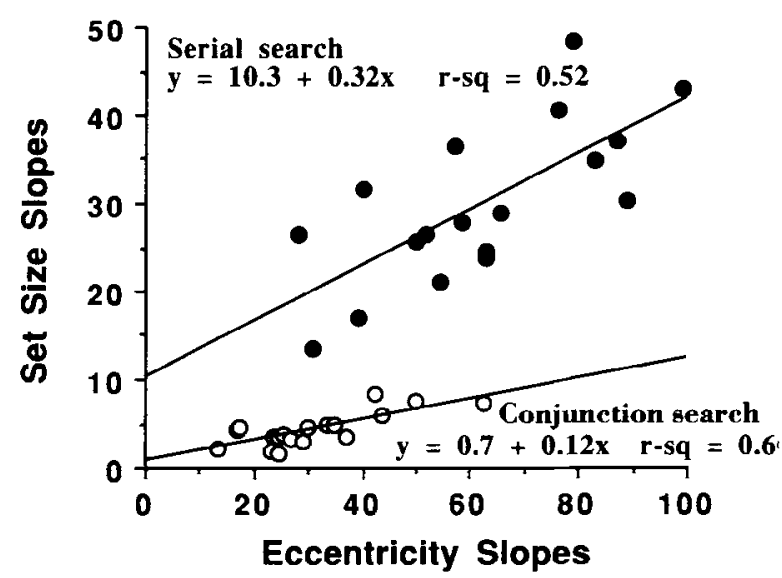

Figure 15. The relationship of set size slopes and eccentricity functions for the two conditions of Experiment 6.

search would be required to say anything definitive on this point.

\section{GENERAL DISCUSSION}

To summarize the results, Experiment 1 replicated the basic eccentricity effect experiments of Carrasco et al. (1995), confirming that there is a substantial effect to explain. Experiments 2, 3, and 4 provided evidence against various explanations that invoke purely visual processes without an attentional component. Eccentricity effects were much reduced when the set size was 1 (Experiment 2 ). Manipulation of crowding produced only a small difference (Experiment 3). Scaling in accord with the cortical magnification factor does not necessarily eliminate eccentricity functions in visual search (Experiments 4 and 5). We propose that eccentricity functions reflect a bias in the deployment (or allocation) of attention to items in the display. Items near fixation receive more attention than do those in the periphery. When the items near fixation were eliminated by having all items in one display presented at one eccentricity, we found that the effects of target eccentricity were largely eliminated (Experiment 5). Finally, the attentional account of eccentricity effects predicts that the slopes of RT $\times$ set size functions should increase as the eccentricity of the targets increases. The results of Experiment 6 showed this to be the case.

Although this paper focused on the effects of eccentricity, it should be remembered that azimuth has an effect, too (Ahissar \& Hochstein, 1994; Bryden \& Mondor, 1991; Christman \& Naegele, 1995; Efron, Yund, \& Nichols, 1987, 1990; Previc \& Blume, 1993; Yund et al., 1990). The general finding seems to be that upper fields are better than lower, and right fields are better than left. There may be sex differences (Ahissar \& Hochstein, 1994; Efron et al., 1987); however, at this stage, it may be safer to note that there are large individual differences of some sort in the pattern of attentional bias. This raises the possibility that the precise form of an individual's eccentricity function is a product of experiences and of hard- wired properties of the visual system. There is evidence, for example, that the UFOV can be expanded with training (Ball et al., 1988). We attempted to bias eccentricity functions by placing the bulk of targets at restricted loci in the display (e.g., presenting the majority of targets in vertical midline locations). We did obtain some evidence for modulation of eccentricity functions by such manipulations but more work is needed.

To reiterate a point made at the outset of this paper, the investigation of eccentricity effects is of more than purely theoretical interest. Many perceptual tasks cannot be done without deployment of attention. The data on eccentricity effects show that this deployment can be slowed by hundreds of milliseconds by manipulations of task and subject variables. Any task that requires a decision in a relatively short amount of time could feel the impact of eccentricity effects. It is, therefore, important not to ignore these effects in our efforts to understand visually guided behavior.

\section{REFERENCES}

Ahissar, M., \& Hochstein, S. (1994). Spatial anisotropy in feature search performance and learning. Investigative Opthalmology \& $\mathrm{Vi}$ sual Science, 35, 2080.

Ball, K. K., Beard, B. L., Roenker, D. L., Miller, R. L., \& Griggs, D. S. (1988). Age and visual search: Expanding the useful field of view. Journal of the Optical Society of America A, 5, 2210-2219.

Ball, K. K., Owsley, C., \& Beard, B. (1990). Clinical visual field perimetry underestimates visual field problems in older adults. Clinical Vision Science, 5, 113-125.

Ball, K. K., Owsley, C., Sloane, M. E., Roenker, D. L., \& Bruni, J. R. (1993). Visual attention problems as a predictor of vehicle crashes among older drivers. Investigative Ophthalmology \& Visual Science, 34, 3110-3123.

BALl, K. K., Roenker, D. L., \& BRUNi, J. R. (1990). Developmental changes in attention throughout adulthood. In J. T. Enns (Ed.), The development of attention: Research and theory (pp. 489-508). Amsterdam: Elsevier.

BennetT, S. C., \& Wolfe, J. M. (1996). Serial search can proceed at $50 \mathrm{msec}$ per item. Investigative Opthalmology \& Visual Science, 37, \$298.

Bryden, M. P., \& Mondor, T. A. (1991). Attentional factors in visual field asymmetries. Canadian Journal of Psychology, 45, 427-447.

BURSILL, A. E. (1958). The restriction of peripheral vision during exposure to hot and humid conditions. Quarterly Journal of Experimental Psychology, 10, 113-129.

Carrasco, M., \& Chang, I. (1995). The interaction of objective and subjective organizations in a localization search task. Perception \& Psychophysics, 57, 1134-1150.

Carrasco, M., \& Evert, D. L. (1991, November). The effect of target position in a conjunction visual search task. Paper presented at the annual meeting of the Psychonomic Society, San Francisco.

Carrasco, M., Evert, D. L., Chang, I., \& Katz, S. M. (1995). The eccentricity effect: Target eccentricity affects performance on conjunction searches. Perception \& Psychophysics, 57, 1241-1261.

Carrasco, M., \& FrIeder, K. S. (1997). Cortical magnification neutralizes the eccentricity effect in visual search. Vision Research, 37, 63-82.

Carrasco, M., \& Katz, S. (1992, April). The effect of target position in a feature visual search task. Paper presented at the annual meeting of the Eastern Psychological Association, Boston.

Carrasco, M., Mclean, T. L., \& Frieder, K. S. (in press). Magnifying spatial frequency and orientation eliminates the eccentricity effect found in visual search. Vision Research.

Christman, S., \& NaEgele, P. (1995, November). Visual search as a function of retinal locus of target. Paper presented at the annual meeting of the Psychonomic Society, Los Angeles.

Chun, M. M., \& Wolfe, J. M. (1996). Just say no: How are visual 
searches terminated when there is no target present? Cognitive Psychology, 30, 39-78.

COHEN, A., \& IVRY, R. B. (1989). Illusory conjunction inside and outside the focus of attention. Journal of Experimental Psychology: Human Perception \& Performance, 15, 650-663.

Cole, B. L., \& Hughes, P. K. (1984). A field trial of attention and search conspicuity. Human Factors, 26, 299-313.

DRASDO, N. (1991). Neural substrates and threshold gradients of peripheral vision. In J. J. Kulikowski, V. Walsh, \& I. J. Murray (Eds.), Limits of vision (Vol. 5, pp. 251-265). Boca Raton, FL: CRC.

DUNCAN, J., \& HumphrEYS, G. W. (1989). Visual search and stimulus similarity. Psychological Review, 96, 433-458.

DunCan, J., Ward, R., \& Shapiro, K. (1994). Direct measurement of attention dwell time in human vision. Nature, 369, 313-314.

EFron, R., Yund, E. W., \& NichOLs, D. R. (1987). Scanning the visual fields without eye movements-A sex difference. Neuropsychologia, 25, 637-644.

EFron, R., Yund, E. W., \& Nichols, D. R. (1990). Serial processing of visual spatial patterns in a search paradigm. Brain \& Cognition, 12, 17-41.

EGETH, H. (1977). Attention and preattention. In G. H. Bower (Ed.), The psychology of learning and motivation (Vol. 11, pp. 277-320). New York: Academic Press.

FIoRENTINI, A., \& BeraRDI, N. (1991). Limits in pattern discrimination: Central and peripheral factors. In J. J. Kulikowski, V. Walsh, \& I. J. Murray (Eds.), Limits of vision (Vol. 5, pp. 266-276). Boca Raton, FL: CRC.

GeIsLER, W. S., \& CHOU, K.-L. (1995). Separation of low-level and high-level factors in complex tasks: Visual search. Psychological Review, 102, 356-378.

Graves, M. A., Ball, K. K., Cissell, G. M., West, R. E., Whorely, K., \& EDWARDS, J. D. (1993). Auditory distraction results in functional visual impairment for some older drivers. Investigative Ophthalmology \& Visual Science, 34, 1418.

GrigSBY, S. S., \& Tsou, B. H. (1994). Grating and flicker sensitivity in the near and far periphery. Naso-temporal asymmetries and binocular summation. Vision Research, 34, 2841-2848.

He, S., Cavanagh, P., \& Intriligator, J. (1996). Attentional resolution and the locus of visual awareness. Nature, 383, 334-337.

HunT, S. M. J. (1994). MacProbe: A Macintosh-based experimenter's workstation for the cognitive sciences. Behavior Research Methods, Instruments, \& Computers, 26, 345-351.

IKEDA, M., \& TAKEUCHI, T. (1975). Influence of foveal load on the functional visual field. Perception \& Psychophysics, 18, 255-260.

KINCHLA, R. A. (1974). Detecting target elements in multielement arrays: A confusability model. Perception \& Psychophysics, 15, 149-158.

LEE, D., JuNG, E. S., \& CHUNG, M. K. (1992). Isoresponse time regions for the evaluation of visual search performance in ergonomic interface models. Ergonomics, 35, 243-252.

LeVi, D. M., Klein, S. A., \& Aitsebaomo, A. P. (1985). Vernier acuity, crowding and cortical magnification. Vision Research, 25, 963-977.

LoGAN, G. (1978). Attention in character classification: Evidence for the automaticity of component stages. Journal of Experimental Psychology: General, 107, 32-63.

MADDEN, D. J. (1992). Selective attention and visual search: Revision of an allocation model and application to age differences. Journal of Experimental Psychology: Human Perception \& Performance, 18, 821-836.

MORdKOFF, J. T., \& EGETH, H. (1993). Response time and accuracy revisited: Converging support for the interactive race model. Journal of Experimental Psychology: Human Perception \& Performance, 19, 981-991

Nakayama, K., \& Silverman, G. H. (1986). Serial and parallel processing of visual feature conjunctions. Nature, 320, 264-265.

PAlmer, J. (1995). Attention in visual search: Distinguishing four causes of a set size effect. Current Directions in Psychological Science, 4, 118-123

Previc, F. H., \& Blume, J. L. (1993). Visual search asymmetries in three-dimensional space. Vision Research, 33, 2697-2704.

RatClifF, R. (1978). A theory of memory retrieval. Psychological Review, 85, 59-108.

Remington, R., \& Williams, D. (1986). On the selection and evaluation of visual display symbology: Factors influencing search and identification times. Human Factors, 28, 407-420.
Rovamo, J., \& VIRSU, V. (1979). An estimation and application of the human cortical magnification factor. Experimental Brain Research, 37, 495-510.

SAARINEN, J. ( I993). Shifts in visual attention at fixation and away from fixation. Vision Research, 33, 1113-1117.

SAGI, D., \& JULESZ, B. (1985). Fast noninertial shifts of attention. Spatial Vision, 1, 141-149.

SANDERS, A. F. (1970). Some aspects of the selective process in the functional visual field. Ergonomics, 13, 101-117.

SANDERS, A. F. (1993). Processing information in the functional visual field. In G. d'Ydewalle \& J. Van Rensbergen (Eds.), Perception and cognition: Advances in eye movement research (pp. 3-22). Amsterdam: Elsevier.

SANDERS, A. F., \& BRück, R. (1991). The effect of presentation time on the size of the visual lobe. Bulletin of the Psychonomic Society, 29, 206-208.

SCHWARTz, E. L. (1977). Spatial mapping in primate sensory projection: Analytic structure and relevance to perception. Biological $C y$ bernetics, 25, 181-194.

Scialfa, C. T., Kline, D. W., \& Lyman, B. J. (1987). Age differences in target identification as a function of retinal location and noise level: Examination of the useful field of view. Psychology \& Aging, 2, 14-19.

ToET, A., \& LEVI, D. M. (1992). The two-dimensional shape of spatial interaction zones in the parafovea. Vision Research, 32, 1349-1357.

TownSEND, J. T. (1971). A note on the identifiability of parallel and serial processes. Perception \& Psychophysics, 10, 161-163.

TOWNSEND, J. T. (1976). Serial and within-stage independent parallel model equivalence on the minimum completion time. Journal of Mathematical Psychology, 14, 219-239.

TownSEND, J. T. (1990). Serial and parallel processing: Sometimes they look like Tweedledum and Tweedledee but they can (and should) be distinguished. Psychological Science, 1, 46-54.

Treisman, A. (1993). The perception of features and objects. In A. Baddeley \& L. Weiskrantz (Eds.), Attention: Selection, awareness, and control (pp. 5-35). Oxford: Oxford University Press, Clarendon Press.

Treisman, A., \& Gelade, G. (1980). A feature-integration theory of attention. Cognitive Psychology, 12, 97-136.

Treisman, A., \& Sato, S. (1990). Conjunction search revisited. Journal of Experimental Psychology: Human Perception \& Performance, 16, 459-478.

Treisman, A. M., \& Schmidt, H. (1982). Illusory conjunctions in the perception of objects. Cognitive Psychology, 14, 107-141.

Weltman, G., \& Egstrom, G. H. (1966). Perceptual narrowing in noviçe divers. Human Factors, 8, 499-506.

Williams, L. J. (1982). Cognitive load and the functional field of view. Human Factors, 24, 683-692.

Williams, L. J. (1985). Tunnel vision induced by a foveal load manipulation. Human Factors, 27, 221-227.

Wilson, H. R., Levi, D., Maffei, L., Rovamo, J., \& DeValois, R. (1990). The perception of form: Retina to striate cortex. In L. Spillman \& J. S. Werner (Eds.), Visual perception: The neurophysiological foundations (pp. 231-272). San Diego: Academic Press.

WOLFE, J. M. (1992). "Effortless" texture segmentation and "parallel" visual search are not the same thing. Vision Research, 32, 757-763.

WolfE, J. M. (1994). Guided Search 2.0: A revised model of visual search. Psychonomic Bulletin \& Review, 1, 202-238.

Wolfe, J. M., CAVE, K. R., \& FRAnZEL, S. L. (1989). Guided Search: An alternative to the feature integration model for visual search. Journal of Experimental Psychology: Human Perception \& Performance, 15, 419-433.

Wolfe, J. M., \& GaNCARz, G. (1996). Guided Search 3.0: A model of visual search catches up with Jay Enoch 40 years later. In V. Lakshminarayanan (Ed.), Basic and clinical applications of vision science, (pp. 189-192). Dordrecht: Kluwer.

Yund, E. W., EFron, R., \& Nichols, D. R. (1990). Detectability gradients as a function of target location. Brain \& Cognition, 12, 1-16.

ZOHARY, E., \& HochSTEIN, S. (1989). How serial is serial processing in vision? Perception, 18, 191-200.

(Manuscript received February 5, 1996 ; revision accepted for publication January $8,1997$. 